\title{
Publisher's Note: Electrical measurement of magnetic-field-impeded polarity switching of a ferromagnetic vortex core [Phys. Rev. B 94, 100402(R) (2016)]
}

\author{
Manu Sushruth, Jasper P. Fried, Abdelmadjid Anane, Stephane Xavier, Cyrile Deranlot, Mikhail Kostylev, \\ Vincent Cros, and Peter J. Metaxas
}

(Received 26 September 2016; published 30 September 2016)

DOI: 10.1103/PhysRevB.94.099904

This paper was published online on 8 September 2016 with a typographical error in the author list. The fifth author's name should read as "Cyrile Deranlot." The author name has been corrected as of 27 September 2016. The author name is correct in the printed version of the journal. 